\title{
Well-constrained Completion and Decomposition for Under-constrained Geometric Constraint Problems
}

\author{
Xiao-Shan Gao \\ KLMM, Institute of Systems Science, AMSS, Academia Sinica \\ Gui-Fang Zhang \\ School of Sciences, Beijing Forestry University, Beijing 100083, China
}

\begin{abstract}
In this paper, we consider the optimal well-constrained completion problem, that is, for an under-constrained geometric constraint problem, add automatically new constraints in such a way that the new geometric constraint problem $G$ is well-constrained and the set of equations need to be solved simultaneously in order to solve $G$ has the smallest size. We propose a polynomial time algorithm which gives a partial solution to the above problem.
\end{abstract}

Keywords. Geometric constraint solving, under-constrained

\section{Introduction}

Geometric constraint solving (abbr. GCS) is one of the key techniques in intelligent and parametric $\mathrm{CAD}$, which allows the user to make modifications to existing designs by changing parametric values. GCS methods may also be used in other fields like computer vision, molecular modelling, robotics, and feature-based design. There are four major approaches to GCS: the numerical approach, the symbolic approach, the rule-based approach, and the graph-based approach.

Most existing GCS methods assumed that the problems are well-constrained. This paper will focus on using graph-based algorithms to solve under-constrained problems. The reason behind this research is that a natural way to draw a design figure is to do it incrementally, that is, to add geometric primitives and geometric constraints one by one. After a new primitive or a new constraint is added, it would be better for the constraint solving system to generate the design diagrams. But, before all the necessary constraints are added, the constraint problem is under-constrained.

Joan-Arinyo et al proposed that the main problem about under-constrained problems are well-constrained completion and completion[12]. For the definition of under- and wellconstrained problem, please see Section 2.1

Problem 1.1 [Well-constrained Completion] Given the geometric constraint graph associated to an under-constrained geometric constraint problem, add automatically new constraints to the graph in such a way that the corresponding geometric constraint problem is well-constrained.

Problem 1.2 [Completion] Given the geometric constraint graph associated to an underconstrained geometric constraint problem, add automatically new constraints to the graph in 
such a way that the corresponding geometric constraint problem is solvable (i.e. the corresponding geometric constraint problem can be solved by construction techniques).

Since not all under-constrained problems have solvable completions, we may further propose the following more general problem.

Problem 1.3 [Optimal Well-constrained Completion] Given the geometric constraint graph associated to an under-constrained geometric constraint problem, add automatically new constraints to the graph in such a way that the corresponding geometric constraint problem $G$ is well-constrained and the set of equations need to be solved simultaneously in order to solve $G$ has the smallest size.

Latham and Middleditch[14] gave a method to detect whether the constraint graph in $2 \mathrm{D}$ or $3 \mathrm{D}$ is over- or under-constrained and decide how to delete or add constraints to make it well-constrained according to the constraint priority. Therefore, Latham and Middleditch solved Problem 1.1. But the part on how to add new constraints is not studied in detail. So their method does not address Problems 1.2 and 1.3. Fudos and Hoffmann[3] proposed a method which could handle those problems that can be solved with the so-called cluster formation method. Yuan et al[18] proposed an algorithm based on local propagation. Their algorithm can be used to find the available solutions according to the design intention in certain sense, but cannot deal with constraint problems with looped constraints. Lee et al[16] gave a method to deal with under-constrained problems in 2D by classifying the underconstrained subgraphs into simplified cases and by applying some classification rules. JoanArinyo et al[11, 12] proposed algorithms to solve the well-constrained completion problem and the completion problem in $2 \mathrm{D}$ with the technique of s-tree decomposition. This method can handle those problems that can be solved with the s-tree decomposition method. In this volume, Trombettoni et al[17] used a degree-freedom-analysis to solve under-constrained problems. Their method is polynomial in time but seems cannot generate optimized completions for problems like the one in Figure 10.

In this paper, we will give an improved solution to Problem 1.1 and a partial solution to Problem 1.3. The main ideas of the algorithm are as follows:

First, we observe that fixing the position of several geometric primitives called base primitives could lead to better well-constrained completions. So our algorithm will try to solve the problem starting with a set of base primitives and if we cannot find a decomposition with these base primitives, we will use a new set of base primitives. Since the number of base primitives is less than the number of constraints, this idea leads to a systematical and effective search of optimal well-constrained completions. After a set of base primitives is fixed, we will generate a general construction sequence (GC) (definition in Section 2.) for the under-constrained problem. This sequence gives a solving order for the primitives. So, a natural way to add a new constraint from $o_{1}$ to $o_{2}$ is that $o_{1}$ is before $o_{2}$ in the sequence. In this way, we will not destroy the solving order. Another idea is that when ever possible, we will construct the primitives one by one. We showed that in $2 \mathrm{D}$ case, all the newly added constraints can be used for this purpose. Based on the ideas of base primitives, GC, and constructing single primitives, we give an improved algorithm for Problem 1.1. The complexity of the algorithm is quadratic in terms of the number of the primitives and constraints. 
During the well-constrained completion, we will check whether the well-constrained problem can be split into two smaller problems, and if not, we will select a set of new base primitives and repeat the well-constrained completion again. This part is presented in Section 4. and is an extension of our work in [7]. The final D-tree decomposition method provides an effective way to decompose well- and under-constrained problems. The complexity of the algorithm is quatic in terms of the number of the primitives and constraints.

The main idea of the algorithms presented in this paper could be extended to cover all types of constraints since we use bipartite graphs. The algorithms could also be extended to the 3D case. We will explore these extensions in our future work. In this paper, we limit to ourself to the nontrivial distance and angle constraints.

We only mention distance and angle constraints for simplicity. We pointed out this in section 1 of the paper.

The rest of the paper is organized as follows. In Section 2., we introduce the basic concepts of constraint graph and the method to compute a maximum weighting of a constraint graph. In Section 3., we give an improved method for well-constrained completion. In Section 4., we propose a decomposition method for under- and well-constrained problems. In Section 5 ., we present the conclusions.

\section{Preliminary Notions and Algorithms}

\subsection{Basic Concepts about Constraint Graphs}

In a geometric constraint problem, we consider two types of geometric primitives: points and lines in two dimensional Euclidean plane and two types of geometric constraints: the distance constraint between point/point, point/line and the angular constraint between line/line. In our algorithm, positional constraints will be added. These include to assign a coordinate to a point and the direction to a line. We will use $p_{i}$ and $l_{i}$ to represent points and lines respectively. The angular and distance constraints between two primitives $o_{1}$ and $o_{2}$ are denoted by $\operatorname{ANG}\left(o_{1}, o_{2}\right)=\alpha$ and $\operatorname{DIS}\left(o_{1}, o_{2}\right)=\delta$ respectively.

We use a bipartite graph $G=(V, C, E, \omega)$ or $G=(\mathbf{V}(G), \mathbf{C}(G), \mathbf{E}(G), \omega)$ to represent a geometric constraint problem, where $V$ and $C$ are vertex sets, $E$ is the edge set, $\omega$ defines a weight for each edge. The details are as follows.

1. The vertices in $V$ represent the geometric primitives. For every primitive vertex $v$ in $V, \operatorname{DOF}(v)$ is the degree of freedom (abbr. $\operatorname{DOF}$ ) of $v$, and $\operatorname{DOF}(V)=\Sigma_{v \in V} \operatorname{DOF}(v)$. The DOF of every geometric primitive considered in this paper is two.

2. The vertices in $C$ represent the constraints. For every constraint $c$ of $C, \operatorname{DOF}(c)$ represents the DOF of $c$, i.e. the number of scalar equations to represent the constraint, and $\operatorname{DOF}(C)=\Sigma_{c \in C} \operatorname{DOF}(c)$. Let $H$ be a subgraph of $G$. A constraint vertex in $H$ between two primitives in $H$ is called an internal constraint of $H$. Otherwise, it is called an external constraint of $H$. We use $\operatorname{IDOF}(H)$ to denote the sum of the DOFs of the internal constraints of $H$.

The DOF of the constraint $\operatorname{DIS}\left(p_{1}, p_{2}\right)$ is one if the distance is not zero, otherwise it is two. The DOF of constraint $\operatorname{DIS}(p, l)$ is one. The DOF of constraint $\operatorname{ANG}\left(l_{1}, l_{2}\right)$ is one. 
3. The edges in $E$ are defined as follows: $E=\left\{\left(c, v_{1}\right),\left(c, v_{2}\right) \mid v_{1}, v_{2} \in V, c \in C, c\right.$ is the constraint between $v_{1}$ and $\left.v_{2}\right\}$. A weighting of the bipartite graph $G$ is a non-negative integer associated with each edge, such that the sum of weights of all edges incident at any vertex $o$, denoted by $\omega(o)$, is not more than $\operatorname{DOF}(o)$. The initial weight of each edge is zero. A weighting of a graph is called a maximal weighting if the sum of all the weights in it is not less than the sum for any other weighting of that graph. The edge directions derive from a maximal weighting of $G$. The initial direction of each edge in $E$ is directed from the primitive vertex in $V$ to the constraint vertex in $C$. If its weight is non-zero, it is also directed from the constraint vertex to the primitive vertex.

4. A vertex $v$ is said to be saturated if $\operatorname{DOF}(v)=\omega(v)$. A vertex $v$ that is not saturated is said to be unsaturated and $\operatorname{DOF}(v)-\omega(v)$ is called the degree of unsaturation and is denoted by $\operatorname{DOU}(v)$. It is obvious that $0 \leq \mathrm{DOU}(v) \leq \operatorname{DOF}(v)$. For each vertex $v$ in $G, \operatorname{DEG}(v)$ represents the sum of DOFs of vertices adjacent to $v$.

For example, Figure 1 is a constraint problem and Figure 2 is its bipartite graph.

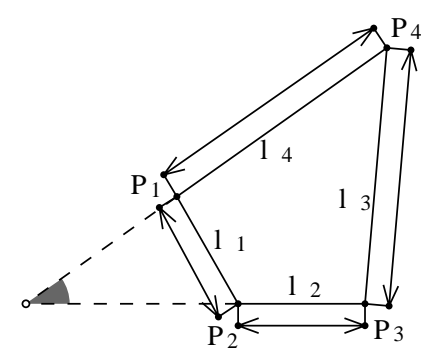

Fig. 1. A constraint problem: Lengths of four edges and $\mathrm{ANG}\left(l_{2}, l_{4}\right)$ are given

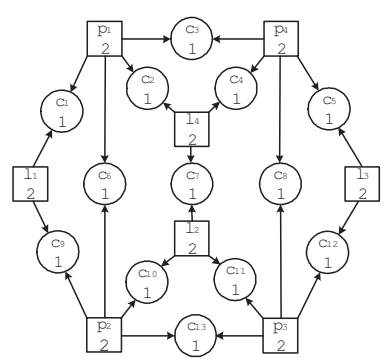

Fig. 2. Graph representation for the problem in Figure 1

The bipartite graph $G$ is called the constraint graph of the geometric constraint problem. A constraint graph $G$ is called (structurally) over-constrained if there is a subgraph $H$ of $G$ such that $H$ contains at least two primitive vertices and satisfies $\operatorname{IDOF}(\mathbf{C}(H))>$ $\operatorname{IDOF}(\mathbf{V}(H))-3$. A constraint graph $G$ is called (structurally) well-constrained if it is not over-constrained and $\operatorname{DOF}(\mathbf{C}(G))=\operatorname{DOF}(\mathbf{V}(G))-3$. A constraint graph $G$ is called (structurally) under-constrained if $G$ is not over-constrained and $\operatorname{DOF}(\mathbf{C}(G))<\operatorname{DOF}(\mathbf{V}(G))-3$.

A constraint system is called geometrically well-constrained if its shape has only a finite number of cases. In most cases, a constraint problem represented by a well-constrained graph $G$ is geometrically well-constrained and hence defines a rigid body. But, in some special cases, a constraint problem represented by a well-constrained graph may have no solutions or an infinite number of solutions. To decide whether a constraint problem is geometrically well-constrained, we need to use techniques from symbolic computation [5]. In this paper, we will focus on the structure analysis of constrained problems and assume that a well-constrained problem defines a rigid body.

\subsection{Computation of the Maximal Weighting}


A maximal weighting of a bipartite graph may be computed by incrementally improving augmenting paths which are pathes directed from an unsaturated primitive to an unsaturated constraint $[8,14]$. Such paths are augmented by adding one to and subtracting one from the weights of alternate edges. The augmentation does not change the sum of weights of edges incident to any interior vertex of the path. It does change the sum of weights for end vertices but the weighting remains valid because the end vertices are unsaturated. For example, diagram (a) in Figure 3 is an augmenting path from $p_{1}$ to $c_{3}$ and diagram (b) is the augmented path.

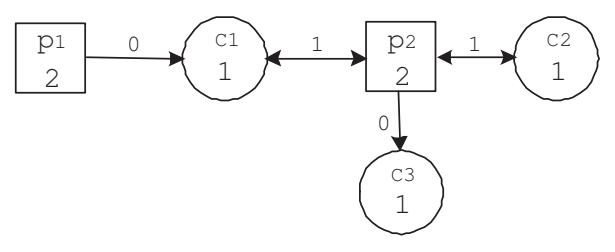

(a)

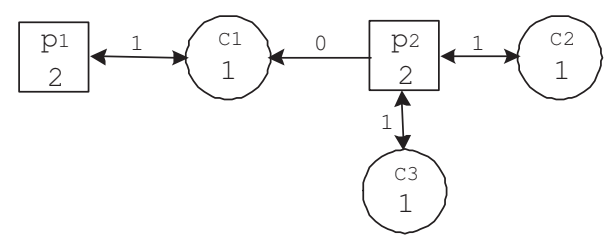

(b)

Fig. 3. Path augmentation

A weighting is maximal when there are no augmenting pathes. Since each augmentation increases the sum of path weights by one, repeated augmentation converts an arbitrary weighting to a maximal weighting. After a constraint is added (or deleted), the augmentation efficiently computes the new maximal weighting from the old maximal weighting. In this case, augmenting paths are easier to find because they include the new constraint[14].

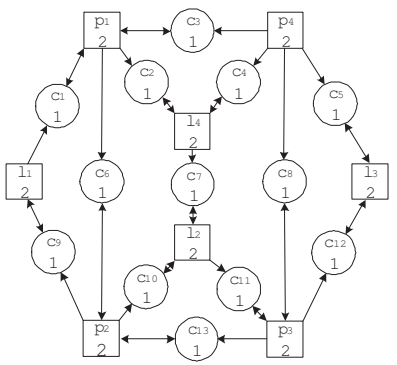

(a)

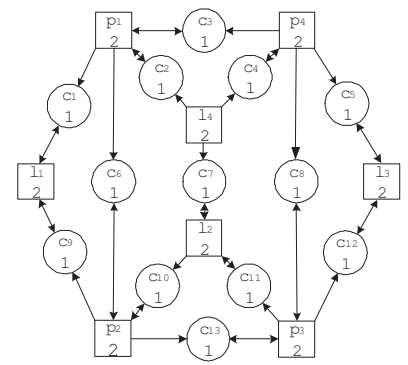

(b)

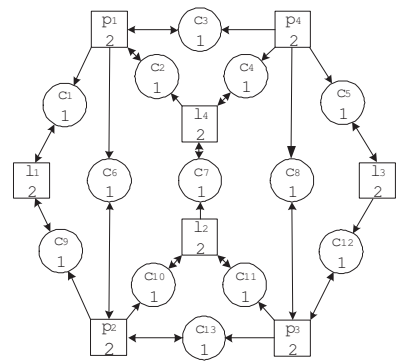

(c)

Fig. 4. Maximal weightings of the problem in Figure 1

There often exist more than one maximal weightings for a constraint graph. For example, in Figure 4, diagrams (a), (b) and (c) are three different maximal weightings for the constraint graph in Figure 1. Diagram (a) has unsaturated primitives $p_{4}$ and $l_{1}$, where $\operatorname{DOU}\left(p_{4}\right)=2$ and $\operatorname{DOU}\left(l_{1}\right)=1$. Diagram (b) has unsaturated primitives $p_{4}$ and $l_{4}$, where $\operatorname{DOU}\left(p_{4}\right)=1$ and $\operatorname{DOU}\left(l_{4}\right)=2$. Diagram (c) has unsaturated primitives $p_{4}$ and $l_{3}$, where $\operatorname{DOU}\left(p_{4}\right)=2$ and $\operatorname{DOU}\left(l_{3}\right)=1$.

In Figure 4, the weight of each edge with single arrowhead is zero, and the weight of each edge with double arrowhead is one.

In a well-constrained or under-constrained graph with a maximal weighting, there exists 
neither unsaturated constraint nor augmenting path, but there exist unsaturated primitives.

\subsection{Partition of a Constraint Graph}

After a maximal weighting of a graph is obtained, the constraint graph will be partitioned into disjoint subgraphs called residual sets. A set of vertices is strongly connected if there is a directed path from any vertex to any vertex in that set. A residual set is a set consisting of only one vertex or a strongly connected set which is not a proper subgraph of another strongly connected set. Residual sets could be computed with graph algorithms[8].

For example, the constraint graph in Figure 4 (a) is partitioned into three residual sets: $\left\{p_{4}\right\},\left\{p_{1}, c_{1}, c_{3}, l_{1}, c_{9}, p_{2}, c_{6}, c_{13}, p_{3}, c_{8}, c_{11}, l_{2}, c_{7}, c_{10}, l_{4}, c_{2}, c_{4}\right\},\left\{l_{3}, c_{5}, c_{12}\right\}$. The constraint graph in Figure 4 (b) is partitioned into six residual sets: $\left\{l_{4}\right\},\left\{p_{4}, c_{4}\right\},\left\{p_{1}, c_{2}, c_{3}\right\}$, $\left\{p_{2}, c_{6}, c_{10}, p_{3}, c_{13}, c_{8}, l_{2}, c_{7}, c_{11}\right\},\left\{l_{3}, c_{5}, c_{12}\right\},\left\{l_{1}, c_{1}, c_{9}\right\}$. The constraint graph in Figure 4 (c) is partitioned into five residual sets: $\left\{p_{4}\right\},\left\{l_{3}, c_{5}\right\},\left\{p_{3}, c_{8}, c_{12}\right\},\left\{p_{1}, c_{2}, c_{3}, p_{2}, c_{6}, c_{13}, l_{2}\right.$, $\left.c_{10}, c_{11}, l_{4}, c_{4}, c_{7}\right\},\left\{l_{1}, c_{1}, c_{9}\right\}$.

Since each directed cycle of the constraint graph with a maximal weighting must lie entirely within a single residual set, and every edge incident to a residual set has a direction, we can impose a partial order to the residual sets according to the directions of the edge connecting them based on Depth First Search. In this paper, we will give a modified procedure to determine the partial order in the next section.

\section{Well-constrained Completion for Under-constrained Problems}

In this section, we will give a solution to the well-constrained completion problem.

\subsection{Find Base Primitives and General Construction Sequences}

The aim of geometric constraint solving is to find the position for the primitives in the problem. For a well-constrained or an under-constrained problem, we need to fix the position of some geometric primitives by adding some new constraints in order to determine the position of the whole diagram. For instance, to determine the position for a triangle whose three sides have known lengths, we need to fix the position of one of its vertices and the direction of one of its sides. These primitives are called base primitives and the newly added positional constraints are called base constraints. For a well-constrained problem $G$, after the base constraints are added, we have $\operatorname{DOF}(\mathbf{C}(G))=\operatorname{DOF}(\mathbf{V}(G))$, and the new problem with these external constraints is called strictly well-constrained.

For well-constrained problems, we may find simpler solutions by selecting specific based primitives[7]. For under-constrained problems, the selection of base primitives is more complicated and more important than the well-constrained case as shown in the rest of this section.

We will try to find a minimal rigid body as shown in Figure 5. This constraint graph represents three types of constraints: $\operatorname{DIS}\left(p_{1}, p_{2}\right)=d(d \neq 0), \operatorname{DIS}(p, l)=d$, and $\operatorname{ANG}\left(l_{1}, l_{2}\right)=$ $a(a \neq 0)$. If such a minimal rigid body does not exist in the constraint problem, we will construct one by adding new primitives. Of course, the construction must not make that the problem becomes over-constrained.

Algorithm 3.1 The input is a constraint graph $G$. The output is a set of base primitives and base constraints. 


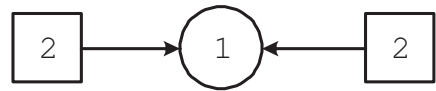

Fig. 5. Minimal rigid bodies

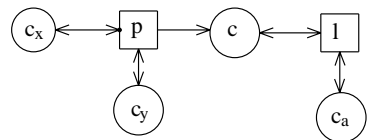

Fig. 6. Base primitives and constraints

1. If there is a distance constraint between a line $l$ and a point $p$, we may fix the position of $p$ and the direction of $l$.

2. If there is a nonzero distance constraint between two points $p_{1}$ and $p_{2}$, we may fix the position of one point and the direction of the line passing through the two points.

3. If there is a non zero angular constraint between two lines $l_{1}$ and $l_{2}$, we may add the intersection point $p$ of $l_{1}$ and $l_{2}$ and fix the position of $p$ and the direction of $l_{1}$.

4. If there exists a line $l$, we may add a new point $p$ on line $l$ and fix the position of $p$ and the direction of $l$.

5. If the conditions in 1-4 are not satisfied, all the geometric primitives must be free points. Let $p_{1}$ and $p_{2}$ be free points. We fix the position of $p_{1}$ and the direction of the line $p_{1} p_{2}$.

The output of Algorithm 3.1 is a constraint problem shown in Figure 6, where $c_{x}, c_{y}, c_{a}$ are the newly added constraints meaning to fix the $x$ coordinate of a point, the $y$ coordinate of a point, the direction of a line respectively. They are new types of constraints and are only used as base constraints. It is clearly that $\left\{p, c_{x}, c_{y}\right\},\left\{l, c, c_{a}\right\}$ are two residual sets. Similar ideas of pinning the problem were also used in [15].

After a set of primitives is selected, we may impose a partial order among the residual sets.

Algorithm 3.2 The input is a set of base primitives $S_{b}$. The output is a sequence of the residual sets with a partial order where the constraint subgraph induced by $S_{b}$ is at the beginning of the sequence.

1. Add the base primitives and constraints to the problem if they are not parts of the problem. Find the residual sets from a maximal weighting of the new constraint graph with methods in Section 2.

2. Let $S_{b}$ be the problem in Figure 6 . Since $c_{x}, c_{y}$ always direct to $p$ and $c_{a}$ always directs to $l$, we cannot change Figure 6 with a path augmentation. Therefore, $\left\{p, c_{x}, c_{y}\right\}$ and $\left\{l, c, c_{a}\right\}$ are two residual sets in the maximal weighting. We select $\left\{p, c_{x}, c_{y}\right\}$ as the first and $\left\{l, c, c_{a}\right\}$ as the second in the sequence of residual sets. Delete all the edges whose tails are in $S_{b}$.

3. Delete a residual set $S$ which has no edges incident to it. This residual set is not connected with other parts of the graph. Repeat this step until no such residual sets exist. 
4. If all the edges incident with $S$ are directed from $S$ to other residual sets, delete $S$ and the edges whose tails are in $S$. Repeat this step until no residual sets exist.

Steps 3 and 4 could be improved with the incremental topological sort [10], which is of linear complexity in terms of the number of residual sets if using a proper data structure.

The order of the deletion is the partial order among the residual sets. We thus obtain a sequence of residual sets

$$
\mathcal{G}: S_{1}, S_{2}, \cdots, S_{n},
$$

such that $S_{i} \leq S_{j}$ for $i<j$. $\mathcal{G}$ is called a general construction sequence of the constraint problem. Geometrically, $S_{i},(i=2, \cdots, n)$ will be determined by $S_{1}, \cdots, S_{i-1}$. Therefore, the largest $\operatorname{DOF}\left(\mathbf{V}\left(S_{i}\right)\right)$ for $i=1, \cdots, n$ is the maximal number of simultaneous equations to be solved in order to solve the GC. This number is called the controlling DOF of the GC $\mathcal{G}$ and is denoted by $\operatorname{MDOF}(\mathcal{G})$.

For the problem in Figure 1, there are three essentially different GCs shown in Figure 4 before adding the base primitives:

$$
\begin{aligned}
& \mathcal{G}_{1}:\left\{p_{4}\right\},\left\{p_{1}, c_{1}, c_{3}, l_{1}, c_{9}, p_{2}, c_{6}, c_{13}, p_{3}, c_{8}, c_{11}, l_{2}, c_{7}, c_{10}, l_{4}, c_{2}, c_{4}\right\},\left\{l_{3}, c_{5}, c_{12}\right\} \\
& \mathcal{G}_{2}:\left\{l_{4}\right\},\left\{p_{4}, c_{4}\right\},\left\{p_{1}, c_{2}, c_{3}\right\},\left\{p_{2}, c_{6}, c_{10}, p_{3}, c_{13}, c_{8}, l_{2}, c_{7}, c_{11}\right\},\left\{l_{3}, c_{5}, c_{12}\right\},\left\{l_{1}, c_{1}, c_{9}\right\} \\
& \mathcal{G}_{3}:\left\{p_{4}\right\},\left\{l_{3}, c_{5}\right\},\left\{p_{3}, c_{8}, c_{12}\right\},\left\{p_{1}, c_{2}, c_{3}, p_{2}, c_{6}, c_{13}, l_{2}, c_{10}, c_{11}, l_{4}, c_{4}, c_{7}\right\},\left\{l_{1}, c_{1}, c_{9}\right\} .
\end{aligned}
$$

If using GCs $\mathcal{G}_{1}, \mathcal{G}_{2}$ and $\mathcal{G}_{3}$ to solve the problem, we have $\operatorname{MDOF}\left(\mathcal{G}_{1}\right)=\operatorname{DOF}\left(\left\{p_{1}, l_{1}, p_{2}, p_{3}, l_{2}, l_{4}\right\}\right)=$ $12, \operatorname{MDOF}\left(\mathcal{G}_{2}\right)=\operatorname{DOF}\left(\left\{p_{2}, p_{3}, l_{2}\right\}\right)=6, \operatorname{MDOF}\left(\mathcal{G}_{3}\right)=\operatorname{DOF}\left(\left\{p_{1}, p_{2}, l_{2}, l_{4}\right\}\right)=8$. It is clear that $\mathcal{G}_{2}$ is the best one and $\mathcal{G}_{1}$ is the worst one. If using Algorithm 3.1 to select base primitives, only $\mathcal{G}_{2}$ and $\mathcal{G}_{3}$ in (1) could be generated. In order to find better completions, we could find rigid bodies consisting of three geometric primitives and treat them as base primitives[7]. If using this heuristic, we will only generate $\mathcal{G}_{2}$ in Figure 4.

\subsection{Well-constrained Completion}

Let

$$
S_{1}, S_{2}, \cdots, S_{n}
$$

be a GC generated with Algorithm 3.2. Let $\mathcal{B}_{k}=\cup_{i=1}^{k} S_{i}$ and $\mathcal{U}_{k}=S_{k+1}$. We will call the problem of determining $\mathcal{U}_{k}$ based on $\mathcal{B}_{k}$ a general basic merge pattern (abbr. GBMP). If both $\mathcal{B}_{k}$ and $\mathcal{B}_{k} \cup \mathcal{U}_{k}$ are strictly well-constrained problems, then the GBMP is a basic merge pattern (BMP) introduced in [7].

The sum of DOFs for constraints between primitives in $\mathcal{B}_{k}$ and $\mathcal{U}_{k}$, denoted by $\operatorname{CN}\left(\mathcal{B}_{k}, \mathcal{U}_{k}\right)$, is called the connection number.

Let $\mathcal{U}_{k}=\left(\mathbf{V}\left(\mathcal{U}_{k}\right), \mathbf{C}\left(\mathcal{U}_{k}\right), \mathbf{E}\left(\mathcal{U}_{k}\right), \omega\right)$. Since the problem is under-constrained or wellconstrained and we need exactly $\operatorname{DOF}\left(\mathbf{V}\left(\mathcal{U}_{k}\right)\right)$ constraints to determine $\mathcal{U}_{k}$, we must have

$$
\mathrm{CN}\left(\mathcal{B}_{k}, \mathcal{U}_{k}\right)+\operatorname{IDOF}\left(\mathcal{U}_{k}\right) \leq \operatorname{DOF}\left(\mathbf{V}\left(\mathcal{U}_{k}\right)\right)
$$

Algorithm 3.3 The input is an under-constrained constraint graph $G$ and the output is a well-constrained graph $G^{\prime}$ which contains $G$ as a subgraph.

1. Find a set of base primitives and add the base constraints with Algorithm 3.1. 
2. Find a GC (2) with Algorithm 3.2. Since the base primitives are always like Figure 6 , we have $S_{1}=\left\{p, c_{x}, c_{y}\right\}, S_{2}=\left\{l, c, c_{a}\right\}$, and $S_{1} \cup S_{2}$ is a strictly well-constrained problem.

3 . Let $k=1$.

4. If $k \geq n$, the algorithm terminates. Otherwise, Let $\mathcal{B}_{k}=\cup_{i=1}^{k} S_{i}$ and $\mathcal{U}_{k}=S_{k+1}$, $d_{k}=\operatorname{DOF}\left(\mathbf{V}\left(\mathcal{U}_{k}\right)\right)-\operatorname{CN}\left(\mathcal{B}_{k}, \mathcal{U}_{k}\right)-\operatorname{IDOF}\left(\mathcal{U}_{k}\right)$. Then by $(3), d_{k} \geq 0$. Also $\mathcal{B}_{k}$ is a strictly well-constrained problem.

5. If $d_{k}=0$, then $\mathcal{B}_{k} \cup \mathcal{U}_{k}$ is a strictly well constrained problem. Let $k:=k+1$ and goto Step 4 .

6. Now $d_{k}>0$ and $\mathcal{B}_{k} \cup \mathcal{U}_{k}$ is under-constrained. Add a new constraint between $\mathcal{B}_{k}$ and $\mathcal{U}_{k}$ with Algorithm 3.4. In Algorithm 3.4, $\mathcal{U}_{k}=S_{k+1}$ is changed to a sequence of residual sets. Replace $S_{k+1}$ in (2) with this sequence, and still denote the new sequence as (2). Goto Step 4.

In order to minimize the controlling DOF of the generated GCs, we adopt the following strategies when adding new constraints. Let $(\mathcal{B}, \mathcal{U})$ be a GBMP such that $\mathcal{B} \cup \mathcal{U}$ is underconstrained.

- When ever possible, we will construct primitives in $\mathcal{U}$ one by one.

- We will add more angular constraints if possible, because constraint problems with more angular constraints are more likely to be solved explicitly $[7,6]$.

According to the above strategies, we design the following method of adding new constraints to an under-constrained problem.

Algorithm 3.4 The input is an under-constrained $\operatorname{GBMP}(\mathcal{B}, \mathcal{U})$. The algorithm will add a new constraint between $\mathcal{B}$ and $\mathcal{U}$ to $\mathcal{U}$ and output a new $G C: \mathcal{B}, D_{1}, \ldots, D_{s}$ such that $\mathcal{U}=\cup_{i=1}^{s} D_{i}$.

1. For a primitive $u$ in $\mathcal{U}$, let $\operatorname{EDOF}(u)$ be the number of constraint vertices directed from $\mathcal{B}$ to $u$ and $\operatorname{IDOF}(u)$ the number of constraint vertices directed from $\mathcal{U}$ to $u$. Since $\operatorname{EDOF}(u)+\operatorname{IDOF}(u) \leq \operatorname{DOF}(u)=2, \operatorname{EDOF}(u)$ and $\operatorname{IDOF}(u)$ could be 0,1 or 2. Search all the vertices in $\mathcal{U}$ to find a $u$ with maximal $\operatorname{EDOF}(u)$. Note that $0 \leq d \leq 2$.

2. The case $\operatorname{EDOF}(u)=2$ cannot happen. Because, in this case, $u$ can be determined by $\mathcal{B}$ alone and $u$ should be the only primitive in $\mathcal{U}$. This contradicts to the condition that $(\mathcal{B}, \mathcal{U})$ is under-constrained.

3. If $\operatorname{EDOF}(u)=1$. There exists an external constraint $b_{1}$ from $\mathcal{B}$ to $u$. We will add a new constraint from $\mathcal{B}$ to $u$ to determine $u$.

- Since $\operatorname{IDOF}(u)+\operatorname{EDOF}(u) \leq 2$, we have $\operatorname{IDOF}(u) \leq 1$. If $\operatorname{IDOF}(u)=1$, there is a constraint $c$ directed from $\mathcal{U}$ to $u$. Since $\mathcal{U}$ is strongly connected, there exist unsaturated primitives in $\mathcal{U}$. Set the weight of the edge between $u$ and $c$ to be zero. 
Constraint $c$ becomes unsaturated. Do an augmentation for the augmenting path from an unsaturated primitive $u_{1}$ in $\mathcal{U}$ to $c$. We obtain a new maximal weighting $\mathcal{U}^{\prime}$. The number of the constraints directed to $u$ from $\mathcal{U}^{\prime}$ will be 0 .

- Now $\operatorname{IDOF}(u)=0$ and $\operatorname{EDOF}(u)=1$. We use Algorithm 3.5 to add one new constraint $b_{2}$ from $\mathcal{B}$ directed to $u$. Now there are two constraints directed from $\mathcal{B}$ to $u$, which are denoted by $C_{u}=\left\{u_{1}, u_{2}\right\}$. This means that $u$ can be determined by $\mathcal{B}$. With the terminology of constraint graph, $u$ and the constraint vertices directed from $\mathcal{B}$ to $u$ consist of a strongly connected subgraph. So, we can let $D=\left\{u, C_{u}\right\}$. Partition $\mathcal{U}^{\prime}$ into a sequence of residual sets $H_{1} \leq \ldots \leq H_{l}$ with Algorithm 3.2 with $D$ as the base primitives. Then $H_{1}=D$, because there is no edge directed from $\mathcal{U}^{\prime}$ to $u$. Return $\mathcal{B}, H_{1}, H_{2}, \ldots, H_{l}$.

4. If $\operatorname{EDOF}(u)=0$, there exist no constraints between $\mathcal{B}$ and $\mathcal{U}$. Since $\mathcal{U}$ is underconstrained, there is an unsaturated primitive $u$ in $\mathcal{U}$. Thus $\operatorname{IDOF}(u) \leq 1$. We could add one new constraint from $\mathcal{B}$ to $u$ similar to Step 3 and return $\mathcal{B}, \mathcal{U}$.

Algorithm 3.5 The input is a $\operatorname{GBMP}(\mathcal{B}, \mathcal{U})$ and an unsaturated primitive $u \in \mathcal{U}$. The output is a primitive $b \in \mathcal{B}$ and a constraint between $u$ and $b$.

1. If there exists a constraint between $u$ and a primitive vertex in $\mathcal{B}$, denote this vertex as $a$. We need to consider two cases.

2. If $u$ is a point, we will select a point or a line $b \in \mathcal{B}$ such that $b \neq a$ and add a constraint $\operatorname{DIS}(b, u)$.

3. Let $u$ be a line. If there exists no angular constraints between $\mathcal{B}$ to $\mathcal{U}$, select a line $b \in \mathcal{B}$ such that $b \neq a$ and add a constraint $\operatorname{ANG}(b, u)$. In all other cases, select a point $b \in \mathcal{B}$ such that $b \neq a$ and add a constraint $\operatorname{DIS}(b, u)$. The motivation behind the selection of $b$ is that we will try to add more angular constraints. But, if there exists an angular constraint between $u$ and a primitive in $\mathcal{B}$, then we cannot add an angular constraint in order to avoid angular conflict.

Algorithm 3.3 solves the well-constrained completion problem. This algorithm improves the algorithm proposed in [14] in several aspects. First, by selecting base primitives carefully, we may generate better well-constrained completions. Second, as shown by the algorithm, all the newly added constraints are used to construct geometric primitives one by one. Third, we will try to add more angular constraints if possible. These strategies lead to easier to solve constraint completions.

Let $n$ and $e$ be the numbers of primitive and edge vertices in $G$ in Algorithm 3.3. Step 1 has complexity $O(e)$. In step 2, we need to find a GC, which has complexity[7] $O(n(n+e))$. Steps 3, 4 and 5 are either constant or linear in terms of $n$ and $e$. In Step 6, we need to call Algorithm 3.4. The complexity of Algorithm 3.4 is $O\left(m^{2}\right)(m=|\mathcal{U}|)$ which is the cost to construct the residual sequence in the second part of Step 3. Let the GBMPs considered in Step 6 of Algorithm 3.3 be $\left(\mathcal{B}_{i}, \mathcal{U}_{i}\right)$ and $m_{i}$ the number of primitive vertices in $\mathcal{U}_{i}$. Then $\sum_{i} m_{i} \leq n$. Therefore, the total complexity of the algorithm is $O((n+e) e)+O\left(\sum_{i} m_{i}^{2}\right) \leq$ $O((n+e) e)+O\left(n^{2}\right)=O\left(n e+e^{2}+n^{2}\right)$. 


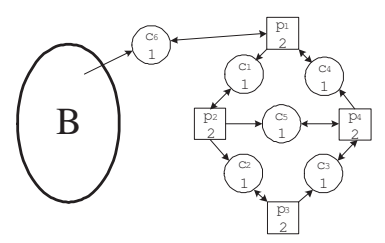

(a)

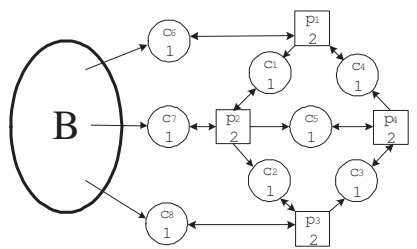

(b)

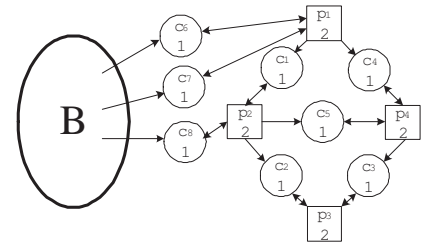

(c)

Fig. 7. An example of adding a new constraint

Diagram (a) in Figure 7 is a GBMP, where $\mathcal{U}$ consists of four points and six nonzero distance constraints. If we add new constraints $c_{7}$ and $c_{8}$ as shown in diagram (b), we must solve a problem of assembling two rigid bodies, which can be reduced to solve three linear equations and one equation of degree six [7]. But we can get an explicit construction sequence $\left\{p_{1}\right\},\left\{p_{2}\right\},\left\{p_{4}\right\},\left\{p_{3}\right\}$ if we add new constraints $c_{7}$ and $c_{8}$ as shown in diagram (c) according to Algorithm 3.5. The key idea is to construct $p_{1}$ first with Algorithm 3.4. Let us explain Algorithm 3.4 with this example.

1. Let $\mathcal{U}=\left\{p_{1}, c_{4}, c_{6}, p_{2}, c_{1} p_{3}, c_{2}, p_{4}, c_{3}, c_{5}\right\}$. In Step 1, we find only one $u$ with largest $\operatorname{EDOF}(u)$, which is $p_{1}$. We have $\operatorname{IDOF}(u)=1$.

2. In Step 3, since $p_{3}$ is an unsaturated primitive, let the weight of the edge between $p_{1}$ and $c_{4}$ becomes zero. Thus $c_{4}$ is an unsaturate constraint vertex, and there is an augmenting path from $p_{3}$ to $c_{4}$. After the augmenting path is augmented, we obtain a new maximal weighting. Add a new external constraint $c_{7}$ between $\mathcal{B}$ and $p_{1}$ to obtain a new graph $U^{\prime}$. Let $\mathcal{B}^{\prime}=\mathcal{B} \cup\left\{p_{1}, c_{6}, c_{7}\right\}$. Find a new maximal weighting for $\mathcal{U}^{\prime}$, which leads to a new GC: $\left\{p_{1}, c_{6}, c_{7}\right\},\left\{p_{2}, c_{1}\right\},\left\{p_{4}, c_{4}, c_{5}\right\},\left\{p_{3}, c_{2}, c_{3}\right\}$.

In the next step, $p_{2}$ will be constructed in a similar way.

Note that the well-constrained completion need not to be unique. The addition of new constraints depend on the selection of base primitives in Algorithm 3.3, the selection of $u$ in Step 1 of Algorithm 3.4, and the selection of $b$ in Algorithm 3.5. These multiple selections can not be avoided in certain sense. For instance, if we need to construct a line $l$, then we could add a distance constraint between $l$ and a point or an angular constraint between $l$ and a line.

\section{Decomposition for Under- and Well-constrained Problem}

In this section, we will propose an algorithm which can be used to find a well-constrained completion for an under-constrained graph and to decompose the well-constrained completion into smaller parts which are represented by a tree.

\subsection{A Decomposition Tree}

Let $G$ be a strictly well-constraint graph and $H$ a strictly well-constrained subgraph of $G$. Let $\mathbf{V}(I)$ be the set of primitive vertices $u \in H$ such that there exists at least one constraint between $u$ and a primitive vertex in $\mathbf{V}(G)-\mathbf{V}(H)$, and $I$ the subgraph of $G$ induced by $\mathbf{V}(I)$. 
If $\mathbf{V}(I) \neq \mathbf{V}(H), H$ is called a faithful subgraph. The importance of a faithful subgraph is that we can use it to reduce the original problem into two smaller ones.

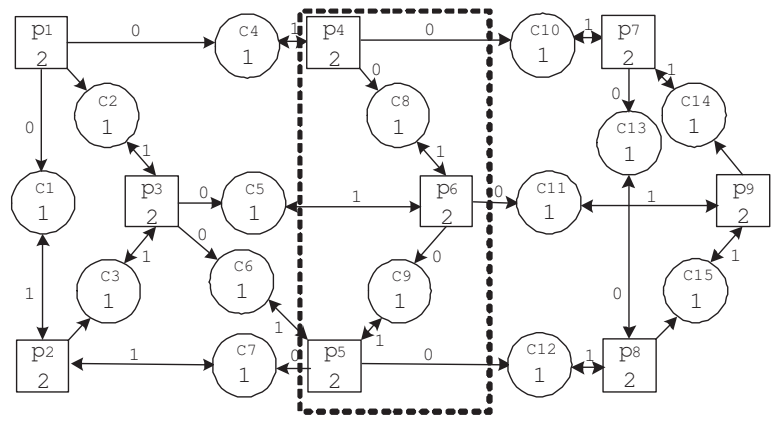

Fig. 8. An example to show the relation between the faithful and split subgraphs.

Let $H$ be a faithful subgraph of a well-constrained graph $G$. We may construct a cut subgraph (called border in [1]) $I$ and a split subgraph $S$ of $G$ with $H$ as follows. Let $I$ be defined as in the preceding paragraph. $I$ could be an under-constrained problem. We may use Algorithm 3.4 to generate a well-constrained problem and still denote it as $I$. This $I$ is called the cut subgraph of $G$ with respect to $H . \mathbf{V}(I)$ is a subset of $\mathbf{V}(H)$, where $H$ is a rigid body. Suppose that $H$ is already solved. Hence the values of the newly added constraints could be computed from the coordinates of the primitives in $H$. Let $\mathbf{V}(S)=(\mathbf{V}(G)-\mathbf{V}(H)) \cup \mathbf{V}(I)$. The subgraph induced by $\mathbf{V}(S)$ is called the split graph of $G$ with respect to $H$. Figure 8 illustrates the relationship between the faithful and split subgraphs, where the subgraph induced by $\left\{p_{4}, p_{5}, p_{6}\right\}$ is the cut graph, the subgraph induced by $\left\{p_{1}, p_{2}, p_{3}, p_{4}, p_{5}, p_{6}\right\}$ is a faithful graph.

A decomposition tree (abbr. D-tree) for a constraint graph $G$ is a ternary tree. The root of the tree is $G$. For each node $N$ in the tree, its left child $L$, middle child $M$ and right child $R$ are as follows.

1. $L$ is a faithful subgraph of $N, M$ is the cut subgraph of $N$ with respect to $L$, and $R$ is the split subgraph of $N$ with respect to $L$; or

2. $N$ is a strictly well-constrained problem and $L$ is a GC for $N, M=\emptyset$ and $R=\emptyset$.

After a D-tree is obtained, we may use it to solve the constraint problem as follows.

Algorithm 4.1 The input is a D-tree T. The outputs are the coordinates of the geometric primitives.

1. We do a left to right depth-first search of the D-tree and consider three cases:

2. The current node $N$ is a GC $\mathcal{G}$ for a strictly well-constrained problem. The problem is reduced to the computation of $\mathcal{G}$ which is discussed in $[6,7]$.

3. The current node $N$ only has a left child $L$. In this case, $L$ is evaluated in Step 2 and $N$ is solved. 
4. The current node $N$ has three children. Due to the depth-first search procedure, we already solved the left child $L$. From $L$, we may compute the numerical values for the new constraints in $M$. With these new constraints, $R$ is also a well-constrained problem. We may solve the right child recursively with this algorithm and merge the left and right children together to compute $N$. To merge $L$ and $R$, we will consider the following cases.

(a) First, since $N$ is well-constrained, $M$ cannot be the set of one primitive. Otherwise, the sub-graph $M \cup R$ will be over-constrained.

(b) If $M$ consists of two parallel lines, the relative position of $L$ and $R$ can not be fixed because there is a translational DOF between $L$ and $R$. In this case, the problem is not a rigid body. Such an example is shown in Figure 9, where the constraints are $\operatorname{DIS}\left(p_{1}, l_{2}\right), \operatorname{DIS}\left(p_{2}, l_{1}\right), \operatorname{DIS}\left(p_{3}, l_{1}\right)$. Since $l_{1} \| l_{2}$, the triangle $p_{1} p_{2} p_{3}$ can still move alone the direction of $l_{1}$.

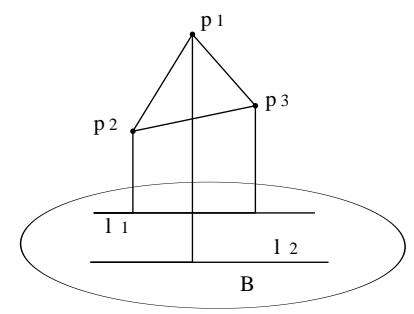

Fig. 9. I consists of two parallel lines

(c) Otherwise, $M$ must contain a minimal rigid body, that is, two points with a nonzero distance constraint, two lines with a non-zero angular constraint, or a point and a line with a distance constraint. In this case, the relative position of $L$ and $R$ can be fixed and $L \cup R$ is a rigid body.

It is clear that the computation of a D-tree is reduced to the computation of GCs. We define the controlling DOF of a D-tree $T, \operatorname{MDOF}(T)$, to be the maximal $\operatorname{MDOF}(C)$ for all general construction sequences $C$ of $T$. A D-tree $T$ for constraint graph $G$ is called minimal if $\operatorname{MDOF}(T)$ is the smallest for all possible D-trees of $G$.

\subsection{An Algorithm to Find a D-tree}

Algorithm 4.2 The input is a well- or under-constrained graph G. The output is a D-tree for $G$.

Set $T=G$ as the initial value.

1. Find a set of base primitives of $T$ with Algorithm 3.1 and still denote the new graph with the base primitives and the base constraints as $T$.

2. Find a GC for $T$ :

$$
\mathcal{G}=S_{1}, \cdots, S_{n}
$$


such that the base primitives appear as the first residual set. Merge those adjacent $S_{i}$ containing only one satured primitive into one set to obtain a reduced $G C$ :

$$
\mathcal{G}^{\prime}=S_{1}^{\prime}, \cdots, S_{m}^{\prime},(m \leq n .)
$$

3. If $m=1$, goto step 7. Otherwise, for $0<k<m$, let $\mathcal{B}_{k}^{\prime}=\cup_{i=1}^{k} S_{i}^{\prime}$ and $\mathcal{U}_{k}^{\prime}=S_{k+1}^{\prime}$.

4. If the problem is under constrained, that is, there is a $k$ such that $\operatorname{CN}\left(\mathcal{B}_{k}, \mathcal{U}_{k}\right)+$ $\operatorname{IDOF}\left(\mathcal{U}_{k}\right)<\operatorname{DOF}\left(\mathbf{V}\left(\mathcal{U}_{k}\right)\right)$, then add one constraint with Algorithm 3.4 between $\mathcal{B}_{k}$ and $\mathcal{U}_{k}$. Still use the notations $\mathcal{G}, S_{i}, \mathcal{B}_{i}, \mathcal{U}_{i}$ for the new problem.

5. Find a minimal $k$ such that $\mathcal{B}_{k}^{\prime} \cup \mathcal{U}_{k}^{\prime}$ is a strictly well-constrained problem and there exists at least one primitive $o \in \mathcal{B}_{k}^{\prime}$ such that there is no constraint between $o$ and primitives in $\mathcal{U}_{k}^{\prime}$. If such a $k$ does not exist, goto the next step; otherwise goto step 8 .

6. There does not exist a faithful subgraph in $\mathcal{G}$.

- If the problem is still under-constrained, goto Step 4.

- Otherwise, select a new set of base primitives and goto Step 2 until there exist no new base primitives.

7. We will solve $T$ with $\mathcal{G}$. A D-tree for $T$ is generated as follows: the left child of $T$ is $\mathcal{G}$ and the middle and right children are empty sets. The algorithm terminates.

8. $\mathcal{B}_{k}^{\prime}$ induces a faithful subgraph $F=\mathcal{B}_{k}^{\prime}$, a cut subgraph $I$ and a split subgraph $S$. We build the D-tree as follows. The left child of $T$ is $F$; the middle child of $T$ is $I$; the right child of $T$ is $S$. Set $T:=S$, remove the newly added constraints from $T$, and goto Step 1.

Let $n$ and $e$ be the numbers of primitive and edge vertices in $G$. Algorithm 4.2 has two main loops: loop one starting from Step 1 for each new $T$, loop two starting from Step 2 for each set of base primitives. After each loop one is executed, at least one primitive is constructed. So, the number of executions for this step is at most $n$. The number of base primitives is $O(e)$. Therefore, the number of executions for loop two is at most $O(e)$. Similar to the complexity analysis for Algorithm 3.3, we can show that the complexity for each execution of loop two is $O\left(e^{2}+n^{2}+n e\right)$. Therefore, the total complexity of the algorithm is $O\left(n e\left(e^{2}+n^{2}+n e\right)\right.$.

Let $G$ be the graph in Figure 10(a), which is also the root of the D-tree.

- In Step 1 we select $\left\{p_{13}, p_{14}\right\}$ as the base primitives.

- In Step 2, we generate a GC:

$\mathcal{G}_{0}:\left\{p_{13}, p_{14}\right\},\left\{p_{1}, p_{2}, p_{3}, p_{4}, p_{15}, p_{5}, p_{6}, p_{7}, p_{8}, p_{9}, p_{10}, p_{11}, p_{12}, p_{16}, p_{17}, p_{18}\right\}$

where the constraints are omitted.

- In Step 4 , we add a constraint $d=\operatorname{DIS}\left(p_{14}, p_{1}\right)$ and generate a reduced GC:

$\mathcal{G}_{1}:\left\{p_{13}, p_{14}, p_{1}, p_{2}, p_{3}, p_{4}, p_{15}\right\},\left\{p_{5}, p_{6}, p_{7}, p_{8}, p_{9}, p_{10}, p_{11}, p_{12}, p_{16}, p_{17}, p_{18}\right\}$ 


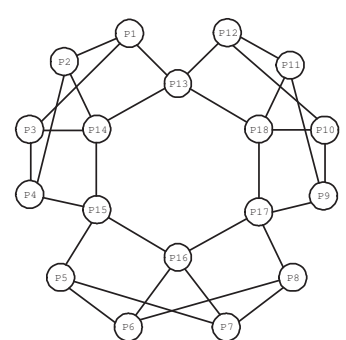

(a)

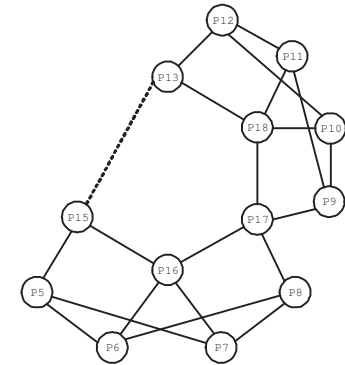

(b)

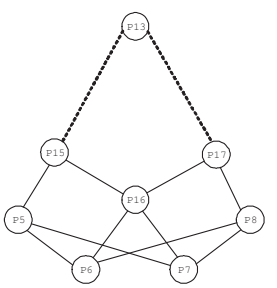

(c)

Fig. 10. Well-constrained completion for an under-constraint problem

- In Step 5, we find a $k=1$. In Step 8, we generate a left child $\mathcal{G}_{2}$ : $\mathcal{G}_{2}:\left\{p_{13}, p_{14}, p_{1}, p_{2}, p_{3}, p_{4}, p_{15}\right\}$.

The cut graph is $\left\{p_{13}, p_{15}\right\}$. A new constraint $\operatorname{DIS}\left(p_{13}, p_{15}\right)$ is added to make the cut graph a well-constrained one. The split graph is the problem in Figure 10 (b). Let $T$ be the split graph. Go back to Step 1.

- Now $T$ is the problem in Figure 10 (b). In Steps $1-4$, select $\left\{p_{17}, p_{18}\right\}$ as the base primitives, add a new constraint $d=\operatorname{DIS}\left(p_{9}, p_{18}\right)$, and generate a new reduced GC:

$\mathcal{G}_{3}:\left\{p_{17}, p_{18}, p_{9}, p_{10}, p_{11}, p_{12}, p_{13}\right\},\left\{p_{5}, p_{6}, p_{7}, p_{8}, p_{15}, p_{16}, p_{17}\right\}$.

- In Step 5, we find a $k=1$. In Step 8, we generate a left child $\mathcal{G}_{4}$ :

$\mathcal{G}_{4}:\left\{p_{17}, p_{18}, p_{9}, p_{10}, p_{11}, p_{12}, p_{13}\right\}$.

A new constraint $\left\{p_{13}, p_{17}\right\}$ is added to make the cut graph a well-constrained one. The split graph is the problem in Figure 10 (c). Go back to Step 1.

- Now $T$ is the problem in Figure 10 (c). Select $\left\{p_{7}, p_{16}\right\}$ as the base primitives. We add a new constraint $d=\operatorname{DIS}\left(p_{16}, p_{8}\right)$ and generate a new general construction sequence:

$\mathcal{G}_{5}:\left\{p_{7}, p_{16}, p_{8}, p_{6}, p_{5}, p_{15}, p_{17}, p_{13}\right\}$

which is already an explicit construction sequence for problem $T$. Now the problem is reduced to the solving of three explicit construction sequences $\mathcal{G}_{2}, \mathcal{G}_{4}, \mathcal{G}_{5}$. The D-tree is given in Figure 11.

Figure 12 is an under-constrained problem where each line represents a distance constraint between two points. This problem cannot be handled with the methods in $[3,11]$, because its constraint graph is tri-connected. Using Algorithm 4.2, we may select $p_{1}$ and $p_{2}$ as the base primitives and add a new constraint $\operatorname{DIS}\left(p_{1}, p_{3}\right)$. Then the problem becomes well-constrained and has the following general construction sequence:

$$
\left\{p_{1}\right\},\left\{p_{2}\right\},\left\{p_{3}\right\},\left\{p_{4}\right\},\left\{p_{5}, p_{6}, p_{7}, p_{8}\right\}
$$

where the constraint vertices are omitted. 


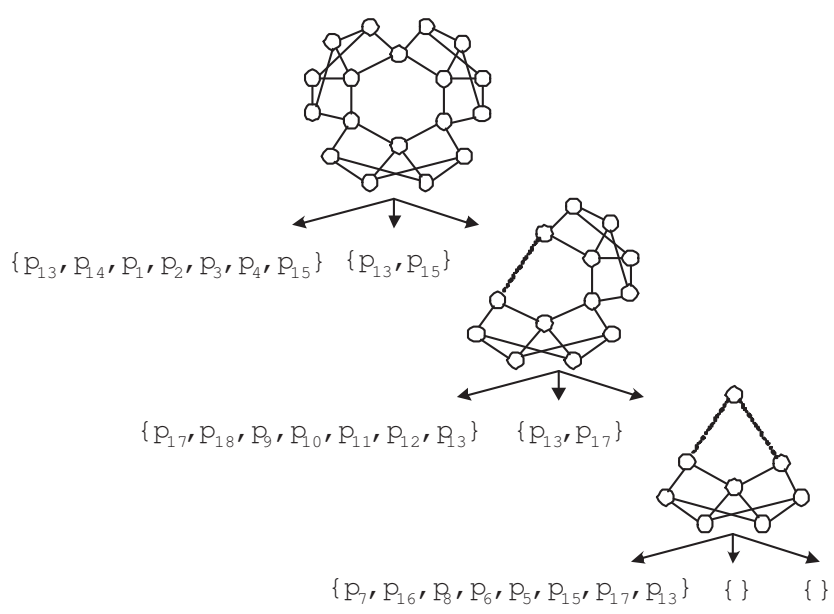

Fig. 11. A D-tree for the problem in Figure 10.

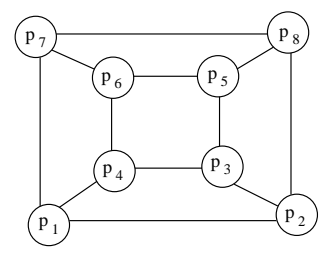

Fig. 12. A tri-connected under-constraint problem

\section{Conclusions}

Handling under-constrained problems is a key and difficult issue in GCS. It is also very useful, because it allows the CAD system to handle constraint problems incrementally. The main difficulty in handling under-constrained problems is how to change it into a wellconstrained problem which is easy to solve, that is, how to find an optimal well-constrained completion.

In this paper, we proposed several strategies and developed algorithms which provide a partial solution to the optimal well-constrained completion problem. The key idea is to use base primitives, GCs, constructing single primitives, and D-tree decompositions. The final D-tree decomposition method provides an effective way to decompose well- and underconstrained problems.

\section{References}

[1] J.F. Dufourd, P. Mathis and P. Schreck, Geometric Construction by Assembling Solved Subfigures, Artificial Intelligence, 99, 73-119, 1998.

[2] C. Durand and C. M. Hoffmann, A Systematic Framework for Solving Geometric Constraints Analytically, J. of Symbolic Computation, 30(5), 493-529, 2000.

[3] I. Fudos and C.M. Hoffmann, A Graph-Constructive Approach to Solving Systems of Geometric Constraints, ACM Trac. on Graphics, 16(2), 179-216, 1997. 
[4] D. Jungnickel, Graphs, Graphen, Netzwerke, und Algorithmen, English Translation, Berlin; Springer, 1999.

[5] X.S. Gao and S.C. Chou, Solving Geometric Constraint Systems, II. A Symbolic Computational Approach, Computer-Aided Design, 30(2), 115-122, 1998.

[6] X.S. Gao, D. Lei, Q. Liao and G. Zhang, Generalized Stewart-Gough Platforms and their Direct Kinematics, IEEE Trans. Robotics, 21(2), 141-151, 2005.

[7] X.S. Gao, Q. Lin and G.F. Zhang, A C-tree Decomposition Algorithm for 2D and 3D Geometric Constraint Solving, Computer-Aided Design, 38,1-13,2006.

[8] M. Gondran and M. Minoux, Graphs and Algorithms, Wiley-Interscience Publications, 1984.

[9] C.M. Hoffmann, A. Lomonosov and M. Sitharam, Decomposition Plans for Geometric Constraint Systems, I: Performance Measures for CAD, 31, 367-408; II: New Algorithms, 31, 409-427, J. of Symbolic Computation, 2001.

[10] R. Hoover, Increamental Graph Evaluation, thesis, Cornell University, Ithaca, 1987.

[11] R. Joan-Arinyo, A. Soto-Riera, S. Vila-Marta and J. Vilaplana-Pastó, Transforming an Underconstrained Geometric Constraint Problem into a Well-constrained one, Proc. ACM SM03, 33-44, ACM Press, New York, 2003.

[12] R. Joan-Arinyo, A. Soto-Riera and S. Vila-Marta, Tools to Deal with Underconstrained Geometric Constraint Graphs, Workshop on Geometric Constraint Solving 2003, http://www.mmrc.iss.ac.cn/ ascm/ascm03/gcsweb/robert.pdf.

[13] R. Joan-Arinyo, A. Soto-Riera, S. Vila-Marta and J. Vilaplana-Pastó, Revisiting Decomposition Analysis of Geometric Constraint Graphs, Computer-Aided Design, 36(2), 123-140, 2004.

[14] R.S. Latham and A.E. Middleditch, Connectivity Analysis: A Tool for Processing Geometric Constraints, Computer-Aided Design, 28, 917-928, 1996.

[15] H. Lamure and D. Michelucci, Qualitative Study of Geometric Constraints, in Geometric Constraint Solving and Applications, 234-258, Springer, Berlin, 1998.

[16] K.Y. Lee, O.H. Kwon, J.Y. Lee and T.W. Kim, A Hybrid Approach to Geometric Constraint Solving with Graph Analysis and Reduction, Advances in Engineering Software, 34, 103-113, 2003.

[17] G. Trombettoni and M. Wilczkowiak, GPDOF: a Polynomial Algorithm to Decompose Under Constrained Systems: Applications to 3D Model Construction, this volume.

[18] B. Yuan, Y. Yuan, S.M. Hu and J.G. Sun, Strategy on Underconstrained Parametric Design (in Chinese), Chinese Journal of Advanced Software Research, 6(4), 305-310, 1999. 